\title{
There Is a Way to Comprise Half-Integer Eigenvalues for Photon Spin
}

\author{
ShaoXu Ren \\ Institute of Physical Science and Engineering \\ Tongji University, 200092, Shanghai, China \\ Corresponding email: shaoxu-ren@hotmail.com \\ Received 13 July; Accepted 25 July; Published 28 July
}

\begin{abstract}
In this article, an attempt based on Spin Topological Space, STS, to give a reasonable detailed account of the cause of photonic fermionization phenomena of light photon is made.

STS is an unconventional spin space in quantum mechanics, which can be used to account for where the unconventional half-integer spin eigenvalues phenomenon of light photon comes from.

We suggest to dectect the possible existence of photonic one-third-spinization phenomenon of light photon, by using three beams of light photon in interference experiment.

\section{Keywords}

Spin Topological Space, STS, Non-Hermitian matrix, Casimir operator, photonic fermionization phenomena, half-integer spin eigenvalues, one third, one fourth spin eigenvalues of photon spin
\end{abstract}

\section{Introduction}

Kyle E. Ballantine, John F. Donegan and Paul R. Eastham [1] measured the total angular momentum of the beam of light with their interferometer, and observed some curious optical phenomena. They found: the eigenvalues of angular momentum of light photon obviously shifted away from the normal physical values that are ruled by the general axioms accepted in today's quantum mechanics world.

Normal angular momentum quantum numbers of the photon must be integers, in units of the Planck constant $\hbar$ : eigenvalues of spin are $-1 \hbar, 0 \hbar,+1 \hbar$ and eigenvalues of orbital are $0 h, 1 \hbar, 2 \hbar, 3 \hbar, \ldots$

However, as the title of their paper, " There are many ways to spin a photon: Half-quantization of a total optical angular momentum " [1] shows: the experimental data in [1] were half-integer, $+\hbar / 2$ and $-\hbar / 2$, or even may be $+1.5 \hbar$ and $-1.5 \hbar \ldots$ ! It is an important physicial experiment result, and indeed, light photon is boson, however possesses fermionic, spectrum ! curious phenomena... 
This present article, "There is a way to comprise half-integer eigenvalues for photon spin ", is in the frame of Spin Topological Space, STS [2] to consider the contributions of spin effects of light photon, and tries to clear up the cause of the photonic fermionization phenomena, which emerged from the experiment [1].

The contributions of orbital effects of light photon, which show half-integer eigenvalues, could appeal to the mechanism of Non-Hermitian orbital angular momentum $\mathbb{L}_{3}, \mathbb{L}^{2}[3]$.

Normally, in quantum mechanics, different kinds of spin particles possess different dimensional spaces, which are expressed by finite dimensional matrices, and these finite dimensional matrices are all Hermiticity.

According to STS, spin angular momentum $\vec{\pi}(l)$ of particles is expressed by infinite dimensional matrices in three-physical space. The first component $\pi_{1}(l)$ and the second component $\pi_{2}(l)$ are Non-Hermitian matrices; the third component $\pi_{3}(l)$ is Hermitian diagonal matrix. Here, mark " $l$ " indicates the lth generation spin particles, $l=1,2,3, \ldots$

2 Three groups of matrices $\vec{\pi}_{+3,-3}(2), \vec{\pi}_{+2,-1}(1), \vec{\pi}_{+3 / 2,-3 / 2}(1)$ of light photon particle $\vec{\pi}(l)$, which satisfy spin angular momentum commutation relus, play the major role in elaborating the machanism of photonic fermionization phenomena.

$$
\begin{aligned}
\vec{\pi}_{+3,-3}(2) \times \vec{\pi}_{+3,-3}(2) & =i \hbar \vec{\pi}_{+3,-3}(2) \\
\vec{\pi}_{+2,-1}(1) \times \vec{\pi}_{+2,-1}(1) & =i \hbar \vec{\pi}_{+2,-1}(1) \\
\vec{\pi}_{+3 / 2,-3 / 2}(1) \times \vec{\pi}_{+3 / 2,-3 / 2}(1) & =i \hbar \vec{\pi}_{+3 / 2,-3 / 2}(1)
\end{aligned}
$$

Where

$$
\begin{aligned}
\vec{\pi}_{+3,-3}(2) & =\left\{\pi_{1 ;+3,-3}(2), \pi_{2 ;+3,-3}(2), \pi_{3 ;+3,-3}(2)\right\} \\
\vec{\pi}_{+2,-1}(1) & =\left\{\pi_{1 ;+2,-1}(1), \pi_{2 ;+2,-1}(1), \pi_{3 ;+2,-1}(1)\right\} \\
\vec{\pi}_{+3 / 2,-3 / 2}(1) & =\left\{\pi_{1 ;+3 / 2,-3 / 2}(1), \pi_{2 ;+3 / 2,-3 / 2}(1), \pi_{3 ;+3 / 2,-3 / 2}(1)\right\}
\end{aligned}
$$

Or instead of (1), (2), (3), in terms of raising matrix operator $\pi_{j}^{+}$, lowering matrix operator $\pi_{k}^{-}$and $\pi_{3 ; j, k}$, i.e. (7) below, to represent commutation rules (8), (9), (10) of light photon with three different kinds of spin state $(\hbar=1)$ :

$$
\begin{gathered}
\left\{\pi_{j}^{+}(l), \pi_{k}^{-}(l), \pi_{3 ; j, k}(l)\right\} \\
\pi_{+3}^{+}(2) \pi_{-3}^{-}(2)-\pi_{-3}^{-}(2) \pi_{+3}^{+}(2)=2 \pi_{3 ;+3,-3}(2) \\
\pi_{3 ;+3,-3}(2) \pi_{+3}^{+}(2)-\pi_{+3}^{+}(2) \pi_{3 ;+3,-3}(2)=+\pi_{+3}^{+}(2) \\
\pi_{3 ;+3,-3}(2) \pi_{-3}^{-}(2)-\pi_{-3}^{-}(2) \pi_{3 ;+3,-3}(2)=-\pi_{-3}^{-}(2) \\
\pi_{+2}^{+}(1) \pi_{-1}^{-}(1)-\pi_{-1}^{-}(1) \pi_{+2}^{+}(1)=2 \pi_{3 ;+2,-1}(1) \\
\pi_{3 ;+2,-1}(1) \pi_{+2}^{+}(1)-\pi_{+2}^{+}(1) \pi_{3 ;+2,-1}(1)=+\pi_{+2}^{+}(1) \\
\pi_{3 ;+2,-1}(1) \pi_{-1}^{-}(1)-\pi_{-1}^{-}(1) \pi_{3 ;+2,-1}(1)=-\pi_{-1}^{-}(1) \\
\pi_{+3 / 2}^{+}(1) \pi_{-3 / 2}^{-}(1)-\pi_{-3 / 2}^{-}(1) \pi_{+3 / 2}^{+}(1)=2 \pi_{3 ;+3 / 2,-3 / 2}(1) \\
\pi_{3 ;+3 / 2,-3 / 2}(1) \pi_{+3 / 2}^{+}(1)-\pi_{+3 / 2}^{+}(1) \pi_{3 ;+3 / 2,-3 / 2}(1)=+\pi_{+3 / 2}^{+}(1) \\
\pi_{3 ;+3 / 2,-3 / 2}(1) \pi_{-3 / 2}^{-}(1)-\pi_{-3 / 2}^{-}(1) \pi_{3 ;+3 / 2,-3 / 2}(1)=-\pi_{-3 / 2}^{-}(1)
\end{gathered}
$$


Write down the explicit representations of raising matrix operators and lowering matrix operators that appear in the above three formulas (8), (9), (10):

$$
\begin{aligned}
& \pi_{+3}^{+}(2)=\frac{1}{2} \operatorname{diag}\{, 8, \quad 7, \quad 6, \quad 5, \quad 4, \quad 3,2, \quad 1,0, \quad-1,-2, \quad,\}_{+2} \\
& \pi_{-3}^{-}(2)=\frac{1}{2} \operatorname{diag}\{,-2,-1, \quad 0,1,2, \quad 3,4, \quad 5,6,-7,-8,,\}_{-2} \\
& \pi_{+2}^{+}(1)=\operatorname{diag}\{, 7, \quad 6,5,4,3,2, \quad 1, \quad 0,-1, \quad-2,-3,,\}_{+1} \\
& \pi_{-1}^{-}(1)=\operatorname{diag}\{,-4,-3,-2,-1,0,1,2,3,4,5,6,,\}_{-1} \\
& \pi_{+3 / 2}^{+}(1)=\frac{1}{2} \operatorname{diag}\{, 13,11,9, \quad 7, \quad 5, \quad 3,1,-1,-3,-5,-7,,\}_{+1} \\
& \pi_{-3 / 2}^{-}(1)=\frac{1}{2} \operatorname{diag}\{,-7,-5,-3,-1,1,3,5,7,9,11,-13,\}_{-1}
\end{aligned}
$$

Subscripts " +1 " and " -1 " represent the first minor top-right diagonal and the first minor down-left diagonal.

Subscripts " +2 " and " -2 " represent the second minor top-right diagonal and the second minor down-left diagonal.

Subscripts " 0 " indicates major diagonal, sometimes for convenience be omitted.

In condition for keeping photon's Casimir operator invariant, that is, keeping

$$
\begin{aligned}
\pi_{+3,-3}^{2}(2) & =\pi_{+2,-1}^{2}(1)=\pi_{+3 / 2,-3 / 2}^{2}(1)=1(1+1) I_{0} \hbar^{2}=2 I_{0} \hbar^{2} \\
I_{0} & =\operatorname{diag}\{, 1,1,1,1,1,1,1,1,1,1,1,\}_{0}
\end{aligned}
$$

Further, next three groups of math series forms of the spin third component $\pi_{3}(l)$ of light photon are obtained as below

$$
\begin{aligned}
& \pi_{3 ;+3,-3}(2) \\
& =\operatorname{diag}\{, 3 \hbar, 2.5 \hbar, 2 \hbar, 1.5 \hbar, 1 \hbar, 0.5 \hbar, 0 \hbar,-0.5 \hbar,-1 \hbar,-1.5 \hbar,-2 \hbar,,\}_{0} \text { (19) } \\
& \pi_{3 ;+2,-1}(1) \\
& =\operatorname{diag}\{, 6 \hbar, 5 \hbar, 4 \hbar, 3 \hbar, 2 \hbar, 1 \hbar, 0 \hbar,-1 \hbar,-2 \hbar,-3 \hbar,-4 \hbar,,\}_{0}(20) \\
& \pi_{3 ;+3 / 2,-3 / 2}(1) \\
& =\operatorname{diag}\{, 5.5 \hbar, 4.5 \hbar, 3.5 \hbar, 2.5 \hbar, 1.5 \hbar, 0.5 \hbar,-0.5 \hbar,-1.5 \hbar,-2.5 \hbar,-3.5 \hbar,-4.5 \hbar,,\}_{0}
\end{aligned}
$$

(19): Alternating series form of Integer eigenvalues and Half-integer eigenvalues (20): Integer eigenvalues series form

(21): Half-integer eigenvalues series form

(19), (20), (21) are just seperately the figures of what happening in Kyle E. Ballantine's and his colleagues' experiment:

Integer eigenvalues series form (20) and half-integer eigenvalues series form (21) give the accounts of " One family includes have the expected bosonic spectrum with integer eigenvalues, and other family, has a fermionic spectrum, comprising half-integer eigenvalues. " (quoted passage from the paper [1]). 
By the way, (20) $\pi_{3 ;+2,-1}(1)$ and (21) $\pi_{3 ;+3 / 2,-3 / 2}(1)$, both of them are together involved in (19) $\pi_{3 ;+3,-3}(2)$. So it seems that there should exist the third family, alternating series form of Integer eigenvalues and Half-integer eigenvalues (19).

\section{Physical behavior mechanism of photonic fermionization of light photon experiment}

Now, matrices (8), (9), (10) can be used to describe the experiment rusults (17) and (19), (20), (21) of photonic fermionization phenomena of light photon, but from what kind of experimental procedure of physical behavior mechanism, these experimental results arise ?

For this reason, deeper research is given. Be concise, the sign of "(1)", is omitted in follows.

Because $\vec{\pi}_{+2,-1}$ and $\vec{\pi}_{m+2, m-1}$ are spin angular momentums in STS, it means:

$$
\begin{aligned}
\vec{\pi}_{+2,-1} \times \vec{\pi}_{+2,-1} & =i \vec{\pi}_{+2,-1} \\
\vec{\pi}_{m+2, m-1} \times \vec{\pi}_{m+2, m-1} & =i \vec{\pi}_{m+2, m-1}
\end{aligned}
$$

Using the linear combinanation of (2) with (22), a new spin angular momentum $\vec{\pi}_{m / 2+2, m / 2-1}(23)$ is composed, and it obeys commutation rule (24)

$$
\begin{aligned}
& \vec{\pi}_{m / 2+2, m / 2-1}=\frac{1}{2}\left\{\vec{\pi}_{m+2, m-1}+\vec{\pi}_{+2,-1}\right\} \ldots \\
& \vec{\pi}_{m / 2+2, m / 2-1} \times \vec{\pi}_{m / 2+2, m / 2-1}=i \vec{\pi}_{m / 2+2, m / 2-1}
\end{aligned}
$$

$\vec{\pi}_{+2,-1}, \vec{\pi}_{m+2, m-1}$ and $\vec{\pi}_{m / 2+2, m / 2-1}$ all are light photon, since their Carsimir operators equal to $2 \hbar^{2}$, i.e.

$$
\pi_{+2,-1}^{2}=\pi_{m+2, m-1}^{2}=\pi_{m / 2+2, m / 2-1}^{2}=1(1+1) I_{0} \hbar^{2}=2 I_{0} \hbar^{2}
$$

Write down the third component of (23), and its exlicit formulation (26. $\mathrm{m}$ ) as below:

$$
\begin{aligned}
& \pi_{3 ; m / 2+2, m / 2-1}=\frac{1}{2}\left\{\pi_{3 ; m+2, m-1}+\underline{\pi_{3 ;+2,-1}}\right\} ; m=0, \pm 1, \pm 2, \pm 3, \pm 4, \ldots \\
& \Delta \pi_{3 ;+4,+1}=\frac{1}{2}\left\{\pi_{3 ;+6,+3}+\underline{\pi_{3 ;+2,-1}}\right\} \\
& =\operatorname{diag}\{, 8,7,6,5,4,3,2, \quad 1,0,-1,-2,,\} \\
& \diamond \pi_{3 ;+7 / 2,+1 / 2}=\frac{1}{2}\left\{\pi_{3 ;+5,+2}+\underline{\pi_{3 ;+2,-1}}\right\} \\
& =\frac{1}{2} \operatorname{diag}\{, 15,13,11,9,7, \underline{5}, 3,1,-1,-3,-5,,\} \\
& \Delta \pi_{3 ;+3,0}=\frac{1}{2}\left\{\pi_{3 ;+4,+1}+\underline{\pi_{3 ;+2,-1}}\right\} \\
& =\operatorname{diag}\{, 7, \quad 6, \quad 5, \quad 4, \quad 3, \quad 2, \quad 1, \quad 0,-1, \quad-2, \quad-3,,\} \\
& \diamond \pi_{3 ;+5 / 2,-1 / 2}=\frac{1}{2}\left\{\pi_{3 ;+3,0}+\underline{\pi_{3 ;+2,-1}}\right\} \\
& =\frac{1}{2} \operatorname{diag}\{, 13,11,9,7, \quad 5, \quad 3,1,-1,-3,-5,-7,,\} \\
& \pi_{3 ;+2,-1}=\frac{1}{2}\left\{\pi_{3 ;+2,-1}+\underline{\pi_{3 ;+2,-1}}\right\} \\
& =\operatorname{diag}\{, 6,5,4, \quad 3,2, \quad 1,0,-1, \quad-2,-3,-4,,\} \\
& \diamond \pi_{3 ;+3 / 2,-3 / 2}=\frac{1}{2}\left\{\pi_{3 ;+1,-2}+\underline{\pi_{3 ;+2,-1}}\right\} \\
& =\frac{1}{2} \operatorname{diag}\{, \quad 11,9, \quad 7, \quad 5,3, \quad 1,-1, \quad-3,-5, \quad-7,-9, \quad\}
\end{aligned}
$$




$$
\begin{aligned}
& \checkmark \pi_{3 ;+1,-2}=\frac{1}{2}\left\{\pi_{3 ; 0,-3}+\underline{\pi_{3 ;+2,-1}}\right\} \\
& =\operatorname{diag}\{, 5, \quad 4, \quad 3, \quad 2, \quad 1, \quad \underline{0},-1,-2,-3,-4,-5,,\} \\
& \diamond \pi_{3 ;+1 / 2,-5 / 2}=\frac{1}{2}\left\{\pi_{3 ;-1,-4}+\underline{\pi_{3 ;+2,-1}}\right\} \\
& =\frac{1}{2} \operatorname{diag}\{, \quad 9, \quad 7, \quad 5, \quad 3, \quad 1,-1,-3,-5,-7,-9,-11,,\} \\
& \Delta \pi_{3 ; 0,-3}=\frac{1}{2}\left\{\pi_{3 ;-2,-5}+\underline{\pi_{3 ;+2,-1}}\right\} \\
& =\operatorname{diag}\{, 4, \quad 3, \quad 2, \quad 1, \quad 0,-1,-2, \quad-3,-4, \quad-5, \quad-6,\}
\end{aligned}
$$

\section{From (26), two important conclusions are given}

1) There exist two diffrent families of the third component of light photon:

$$
\begin{aligned}
& \text { family BP: Bosonization of Photon, labelled by " ", } \\
& \text { family FP: Fermionization of Photon, labelled by " } \diamond "
\end{aligned}
$$

For light photon, the angular momentum addition of two angular momentums, one angular momentum $\mathbf{B P} 1$ with other angular momentum $\mathbf{F P}_{2}$, may generate two diffrent families of the third component of light photon.

$$
\begin{aligned}
& \mathbf{B P} \triangleleft(m)=\frac{1}{2}\left\{\mathbf{B} \mathbf{P}_{1}(m)+\mathbf{B} \mathbf{P}_{2}(0)\right\} ; m=0, \pm 2, \pm 4, \pm 6, \ldots \\
& \mathbf{F P} \diamond(m)=\frac{1}{2}\left\{\mathbf{B P}_{1}(m)+\mathbf{B P}_{2}(0)\right\} ; m= \pm 1, \pm 3, \pm 5, \pm 7, \ldots
\end{aligned}
$$

$\mathbf{B P} \triangleleft$ and $\mathbf{F P} \diamond$ alternately appear with $m$.

2) For a fixed term of the new spin angular momentum $\pi_{3 ; m / 2+2, m / 2-1}$, there are many options to choose from the general expression (29).

$$
\begin{gathered}
\mathbf{B P} \downarrow\left(m, m^{\prime}\right), \mathbf{F P} \diamond\left(m, m^{\prime}\right)=\frac{1}{2}\left\{\mathbf{B P}_{1}(m)+\mathbf{B P}_{2}\left(m^{\prime}\right)\right\} \\
m, m^{\prime}=0, \pm 1, \pm 2, \pm 3, \ldots
\end{gathered}
$$

Family BP (27) and Family $\mathbf{F P} \diamond$ (28) are the simplest couple, in which one spin angular momentum $\mathbf{B} \mathbf{P}_{2}(0), \underline{\pi_{3 ;+2,-1}}$ is keeping invariant, as other spin angular momentum $\mathbf{B P}_{1}(m), \vec{\pi}_{m+2, m-1}$ varies with $m$ in expression $\pi_{3 ; m / 2+2, m / 2-1}$ (26. $m$ ).

(20) $\pi_{3 ;+2,-1}(1)$ and (21) $\pi_{3 ;+3 / 2,-3 / 2}(1)$, which are the part of expression $\pi_{3 ; m / 2+2, m / 2-1}$ (26. $m$ ). When $m$ equals to 0 and -1 , (20) and (21) are just (26.0) family $\mathbf{B P} \bullet(0)$ and (26.-1) family $\mathbf{F P} \diamond(-1)$.

The relationship between (20) and (21), or equivalent to that between matrices (9) and (10), could refer to the math statements (26.0) and (26.-1). They are the results of the additions of spin angular momentum photon $\pi_{3 ;+2,-1}$ with photon $\underline{\pi_{3 ;+2,-1}}$, and photo $\pi_{3 ;+1,-2}$ with photon $\underline{\pi_{3 ;+2,-1}}$ in Ballantine's and his colleagues' experiment.

By the way, the intervals between two adjoining $\mathbf{B P} \diamond$ and $\mathbf{F P} \diamond$ is $\frac{\hbar}{2}$

$$
\Delta \pi_{3 ; m / 2+2, m / 2-1}=\mathbf{F P} \diamond(m \pm 1)-\mathbf{B P} \downarrow(m)= \pm \frac{\hbar}{2} I_{0}
$$




\section{Prediction about one-third-spinization phenomenon of light photon}

Proceeding in above way, parallel to the math structure of three groups of matrices (11), (12) and (13), (14), (15), (16) for photonic fermionization, we guess at the existent of so-call photonic one-third-spinization phenomenon of light photon, and four groups of matrices (31), (32) and (33), (34), (35), (36), (37), (38) are given, below labelled by " \& ".

$$
\begin{aligned}
& \pi_{+4}^{+}(3)=\frac{1}{2} \operatorname{diag}\{, 9, \quad 8, \quad 7,6, \quad 5,4,3,2, \quad 1,0,-1, \quad,\}_{+3} \\
& \pi_{-5}^{-}(3)=\frac{1}{2} \operatorname{diag}\{, 0,-1,-2,-3,-4,-5,-6,-7,-8,-9,-10,,\}_{-3} \\
& \pi_{+2}^{+}(1)=\operatorname{diag}\{, 7, \quad 6,5, \quad 4,3,2, \quad 1,0,-1,-2,-3,,\}_{+1} \\
& \pi_{-1}^{-}(1)=\operatorname{diag}\{,-4,-3,-2,-1,0,1,2,3,4,5,6,\}_{-1} \\
& \pi_{+5 / 3}^{+}(1)=\frac{1}{3} \operatorname{diag}\{, 20,17,14,11,8, \quad \underline{5}, 2,-1,-4,-7,-10,,\}_{+1} \\
& \pi_{-4 / 3}^{-}(1)=\frac{1}{3} \operatorname{diag}\{, 11,8,5,2,-1, \underline{-4},-7,-10,-13,-16,-19, \quad\}_{-1} \\
& \pi_{+4 / 3}^{+}(1)=\frac{1}{3} \operatorname{diag}\{, 19,16,13,10,7, \underline{4}, 1,-2,-5,-8,-11,,\}_{+1} \\
& \pi_{-5 / 3}^{-}(1)=\frac{1}{3} \operatorname{diag}\{, 10,7,4,1,-2, \underline{-5},-8,-11,-14,-17,-20,\}_{-1}
\end{aligned}
$$

As well as photon's Casimir operator

$$
\pi_{+4,-5}^{2}(3)=\pi_{+2,-1}^{2}(1)=\pi_{+5 / 3,-4 / 3}^{2}(1)=\pi_{+4 / 3,-5 / 3}^{2}(1)=1(1+1) I_{0} \hbar^{2}=2 I_{0} \hbar^{2}
$$

Accordingly, next four groups of math series forms of the spin third component $\pi_{3}(l)$ of light photon are obtained as below

$$
\begin{aligned}
& \pi_{3 ;+4,-5}(3)= \\
& \operatorname{diag}\{, \quad 2, \quad 5 / 3, \quad 4 / 3, \quad 1,2 / 3, \quad \underline{\underline{3}}, \quad 0, \quad-1 / 3,-2 / 3, \quad-1,-4 / 3,\}_{0} \\
& \pi_{3 ;+2,-1}(1)=
\end{aligned}
$$

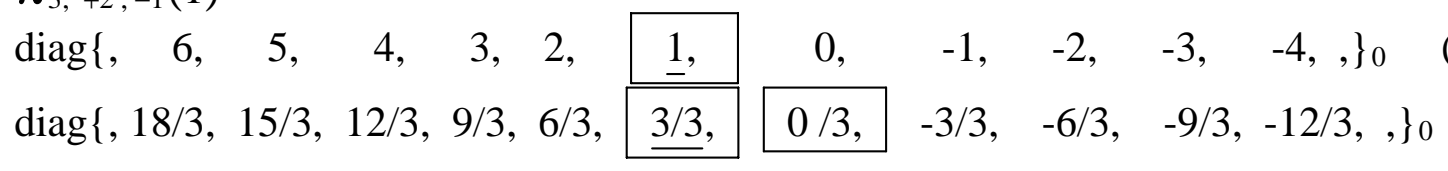

$$
\begin{aligned}
& \pi_{3 ;+5 / 3,-4 / 3}(1)= \\
& \operatorname{diag}\{, 17 / 3,14 / 3,11 / 3,8 / 3,5 / 3,2 / 3, \quad-1 / 3,-4 / 3,-7 / 3,-10 / 3,-13 / 3,\}_{0}(42) \\
& \pi_{3 ;+4 / 3,-5 / 3}(1)= \\
& \operatorname{diag}\{, 16 / 3,13 / 3,10 / 3,7 / 3,4 / 3,1 / 3,-2 / 3,-5 / 3,-8 / 3,-11 / 3,-14 / 3,\}_{0}
\end{aligned}
$$

Let us have some acquaintance with the relationship among (41), (42), (43), by the general formula of addition of spin angular momentum of light photon (44.m).

It is shown that (41), (42), (43) are (44.0), (44.-1), (44.-2), which are parts of general formular (44) below 
General formula of the addition of light photon are given by (44.m)

$\pi_{3 ; m / 3+2, m / 3-1}=\frac{1}{3}\left\{\pi_{3 ; m+2, m-1}+\underline{2 \pi_{3 ;+2,-1}}\right\} ; m=0, \pm 1, \pm 2, \pm 3, \ldots$

$\diamond \pi_{3 ;+4,+1}=\frac{1}{3}\left\{\pi_{3 ;+8,+5}+\underline{2 \pi_{3 ;+2,-1}}\right\}$

$=\operatorname{diag}\{, 8,7,6, \quad 5,4, \quad \underline{3}, \quad 2,1,0,-1,-2,$,$\} \quad (44.6)$

* $\pi_{3 ;+11 / 3,+2 / 3}=\frac{1}{3}\left\{\pi_{3 ;+7,+4}+\underline{2 \pi_{3 ;+2,-1}}\right\}$

$=\frac{1}{3} \operatorname{diag}\{, 23,20,17,14,11, \underline{8}, \quad 5,2,-1,-4,-7, \quad\}$

\& $\pi_{3 ;+10 / 3,+1 / 3}=\frac{1}{3}\left\{\pi_{3 ;+6,+3}+\underline{2 \pi_{3 ;+2,-1}}\right\}$

$=\frac{1}{3} \operatorname{diag}\{, 22,19,16,13,10, \underline{7}, 4,1,-2,-5,-8,$,

$\checkmark \pi_{3 ;+3,0}=\frac{1}{3}\left\{\pi_{3 ;+5,+2}+\underline{2 \pi_{3 ;+2,-1}}\right\}$

$=\operatorname{diag}\{, 7, \quad 6,5, \quad 4, \quad 3, \quad 2, \quad 1,0,-1,-2,-3,$,

\& $\pi_{3 ;+8 / 3,-1 / 3}=\frac{1}{3}\left\{\pi_{3 ;+4,+1}+\underline{2 \pi_{3 ;+2,-1}}\right\}$

$=\frac{1}{3} \operatorname{diag}\{, 20,17,14,11,8, \quad \underline{5}, \quad 2,-1,-4,-7,-10,$,$\} (44.2)$

* $\pi_{3 ;+7 / 3,-2 / 3}=\frac{1}{3}\left\{\pi_{3 ;+3,0}+\underline{2 \pi_{3 ;+2,-1}}\right\}$

$=\frac{1}{3} \operatorname{diag}\{, 19,16,13,10,7, \quad \underline{4}, 1,-2, \quad-5,-8,-11, \quad\}(44.1)$

$\underline{\pi_{3 ;+2,-1}}=\frac{1}{3}\left\{\pi_{3 ;+2,-1}+\underline{2 \pi_{3 ;+2,-1}}\right\}$

$=\operatorname{diag}\{, 6, \quad 5, \quad 4, \quad 3, \quad 2, \quad 1, \quad 0, \quad-1, \quad-2, \quad-3, \quad-4,\},(44.0)$

$=\frac{1}{3} \operatorname{diag}\{, 18,15,12,9,6, \quad \underline{\underline{3}}, \quad, 0-3,-6,-9,-12, \quad\}$

* $\pi_{3 ;+5 / 3,-4 / 3}=\frac{1}{3}\left\{\pi_{3 ;+1,-2}+\underline{2 \pi_{3 ;+2,-1}}\right\}$

$=\frac{1}{3} \operatorname{diag}\{, 17,14,11,8,5, \quad 2,-1,-4, \quad-7,-10,-13,\},(44 .-1)$

* $\pi_{3 ;+4 / 3,-5 / 3}=\frac{1}{3}\left\{\pi_{3 ; 0,-3}+2 \pi_{3 ;+2,-1}\right\}$

$=\frac{1}{3} \operatorname{diag}\{, 16,13,10,7,4, \quad \underline{1},-2,-5,-8,-11,-14,\},(44 .-2)$

$\Delta \pi_{3 ;+1,-2}=\frac{1}{3}\left\{\pi_{3 ;-1,-4}+\underline{2 \pi_{3 ;+2,-1}}\right\}$

$=\operatorname{diag}\{, 5, \quad 4, \quad 3, \quad 2, \quad 1, \quad \underline{0}, \quad-1, \quad-2, \quad-3, \quad-4,-5,\},(44 .-3)$

$* \pi_{3 ;+2 / 3,-7 / 3}=\frac{1}{3}\left\{\pi_{3 ;-2,-5}+\underline{2 \pi_{3 ;+2,-1}}\right\}$

$=\frac{1}{3} \operatorname{diag}\{, 14,11,8, \quad 5,2, \quad-1,-4,-7,-10,-13,-16, \quad\}(44 .-4)$

* $\pi_{3 ;+1 / 3,-8 / 3}(1)=\frac{1}{3}\left\{\pi_{3 ;-3,-6}+\underline{2 \pi_{3 ;+2,-1}}\right\}$

$=\frac{1}{3} \operatorname{diag}\{, 13,10,7, \quad 4,1, \quad-2,-5, \quad-8,-11,-14,-17, \quad\}(44 .-5)$

$\Delta \pi_{3 ; 0,-3}=\frac{1}{3}\left\{\pi_{3 ;-4,-7}+\underline{2 \pi_{3 ;+2,-1}}\right\}$

$=\operatorname{diag}\{, 4,3,2, \quad 1,0, \quad-1,-2, \quad-3,-4, \quad-5,-6,\},(44 .-6)$

$\mathbf{B P}(m)=\frac{1}{3}\left\{\mathbf{B P}_{1}(m)+2 \mathbf{B P}_{2}(0)\right\} ; \quad m=0, \pm 3, \pm 6 ., \ldots$

$\mathbf{D P} \boldsymbol{*}(m)=\frac{1}{3}\left\{\mathbf{B} \mathbf{P}_{1}(m)+2 \mathbf{B} \mathbf{P}_{2}(0)\right\} ; \quad m= \pm 1, \pm 2, \pm 4, \pm 5, \ldots$

$\Delta \pi_{3 ; m / 3+2, m / 3-1}=\mathbf{D P} \boldsymbol{*}(m \pm 1)-\mathbf{B P}(m)= \pm \frac{\hbar}{3} I_{0}$

And $\quad \pi_{+2,-1}^{2}=\pi_{m+2, m-1}^{2}=\pi_{m / 3+2, m / 3-1}^{2}=1(1+1) I_{0} \hbar^{2}=2 I_{0} \hbar^{2}$ 
Combine (44), (26), obtian:

$\pi_{3 ; m / 6+2, m / 6-1}=\frac{1}{3}\left\{\pi_{3 ; m+2, m-1}+\underline{2 \pi_{3 ;+2,-1}}\right\} ; m=0, \pm 1, \pm 2, \pm 3, \ldots$

$$
\begin{aligned}
& \text { - } \pi_{3 ;+4,+1}=\frac{1}{3}\left\{\pi_{3 ;+8,+5}+\underline{2 \pi_{3 ;+2,-1}}\right\} \\
& =\operatorname{diag}\{, \quad 8, \quad 7, \quad 6,5, \quad 4, \quad \underline{3}, \quad 2, \quad 1,0,-1,-2,,\}
\end{aligned}
$$

$\pi_{3 ;+23 / 6,+5 / 6}=\frac{1}{3}\left\{\pi_{3 ;+15 / 2,+9 / 2}+2 \pi_{3 ;+2,-1}\right\}$

$=\frac{1}{6} \operatorname{diag}\{, 47,41,35,29,23, \underline{17}, 11,5,-1,-7,-13$,

$* \pi_{3 ;+11 / 3,+2 / 3}=\frac{1}{3}\left\{\pi_{3 ;+7,+4}+\underline{2 \pi_{3 ;+2,-1}}\right\}$

$=\frac{1}{3} \operatorname{diag}\{, \quad 23,20,17,14,11, \quad \underline{8}, 5,2,-1,-4,-7$,

$\diamond \pi_{3 ;+7 / 2,+1 / 2}=\frac{1}{3}\left\{\pi_{3 ;+13 / 2,+7 / 2}+2 \pi_{3 ;+2,-1}\right\}$

$=\frac{1}{2} \operatorname{diag}\{, \quad 15, \quad 13, \quad 11,9, \quad 7, \quad \underline{5}, 3, \quad 1,-1, \quad-3,-5, \quad\}$

* $\pi_{3 ;+10 / 3,+1 / 3}(1)=\frac{1}{3}\left\{\pi_{3 ;+6,+3}+\underline{2 \pi_{3 ;+2,-1}}\right\}$

$=\frac{1}{3} \operatorname{diag}\{, \quad 22, \quad 19,16,13,10, \quad \underline{7}, \quad 4,1, \quad-2, \quad-5, \quad-8\} \quad,(49.8)$

$\pi_{3 ;+19 / 6,+1 / 6}=\frac{1}{3}\left\{\pi_{3 ;+11 / 2,+5 / 2}+2 \pi_{3 ;+2,-1}\right\}$

$=\frac{1}{6} \operatorname{diag}\{, \quad 43,37,31,25,19, \underline{13}, 7,1,-5,-11,-17$,

$\checkmark \pi_{3 ;+3,0}=\frac{1}{3}\left\{\pi_{3 ;+5,+2}+\underline{2 \pi_{3 ;+2,-1}}\right\}$

$=\operatorname{diag}\{, 7, \quad 6, \quad 5, \quad 4, \quad 3, \quad \underline{2}, \quad 1, \quad 0, \quad-1, \quad-2, \quad-3,$,$\} \quad (49.6)$

$\pi_{3 ;+17 / 6,-1 / 6}=\frac{1}{3}\left\{\pi_{3 ;+9 / 2,+3 / 2}+\underline{2 \pi_{3 ;+2,-1}}\right\}$

$=\frac{1}{6} \operatorname{diag}\{, \quad 41,35,29, \quad 23,17, \underline{11}, 5,-1,-7,-13,-19\} \quad,(49.5)$

* $\pi_{3 ;+8 / 3,-1 / 3}=\frac{1}{3}\left\{\pi_{3 ;+4,+1}+2 \pi_{3 ;+2,-1}\right\}$

$=\frac{1}{3} \operatorname{diag}\{, \quad 20,17, \quad 14, \quad 11,8, \quad \underline{5}, 2, \quad-1,-4, \quad-7,-10$,

$\diamond \pi_{3 ;+5 / 2,-1 / 2}=\frac{1}{3}\left\{\pi_{3 ;+7 / 2,+1 / 2}+2 \pi_{3 ;+2,-1}\right\}$

$=\frac{1}{2} \operatorname{diag}\{, \quad 13, \quad 11, \quad 9, \quad 7, \quad 5, \quad \underline{3}, \quad 1, \quad-1, \quad-3, \quad-5, \quad-7,\} \quad,(49.3)$

$* \pi_{3 ;+7 / 3,-2 / 3}=\frac{1}{3}\left\{\pi_{3 ;+3,0}+\underline{2 \pi_{3 ;+2,-1}}\right\}$

$=\frac{1}{3} \operatorname{diag}\{, \quad 19, \quad 16, \quad 13, \quad 10, \quad 7, \quad 4, \quad 1,-2, \quad-5, \quad-8,-11,\} \quad,(49.2)$

$\pi_{3 ;+13 / 6,-5 / 6}=\frac{1}{3}\left\{\pi_{3 ;+5 / 2,-1 / 2}+\underline{2 \pi_{3 ;+2,-1}}\right\}$

$=\frac{1}{6} \operatorname{diag}\{, 37,31,25, \quad 19,13, \underline{7}, 1,-5,-11,-17,-23$, , $\}$

$\underline{\pi_{3 ;+2,-1}}=\frac{1}{3}\left\{\pi_{3 ;+2,-1}+\underline{2 \pi_{3 ;+2,-1}}\right\}$

$=\operatorname{diag}\{, 6, \quad 5, \quad 4, \quad 3, \quad 2, \quad 1, \quad 0, \quad-1, \quad-2, \quad-3, \quad-4,\} \quad,(49.0)$

$\pi_{3 ;+11 / 6,-7 / 6}=\frac{1}{3}\left\{\pi_{3 ;+3 / 2,-3 / 2}+2 \pi_{3 ;+2,-1}\right\}$

$=\frac{1}{6} \operatorname{diag}\{, \quad 35,29, \quad 23, \quad 17,11, \quad \underline{5},-1,-7,-13,-19,-25,\},(49 .-1)$

$* \pi_{3 ;+5 / 3,-4 / 3}=\frac{1}{3}\left\{\pi_{3 ;+1,-2}+\underline{2 \pi_{3 ;+2,-1}}\right\}$

$=\frac{1}{3} \operatorname{diag}\{, 17, \quad 14, \quad 11, \quad 8, \quad 5, \quad 2,-1,-4, \quad-7, \quad-10,-13,\},(49 .-2)$

$\diamond \pi_{3 ;+3 / 2,-3 / 2}=\frac{1}{3}\left\{\pi_{3 ;+1 / 2,-5 / 2}+\underline{2 \pi_{3 ;+2,-1}}\right\}$

$=\frac{1}{2} \operatorname{diag}\{, \quad 11, \quad 9, \quad 7, \quad 5, \quad 3, \quad 1,-1,-3, \quad-5, \quad-7, \quad-9$, , $\}$ (49.-3) 


$$
\begin{aligned}
& \boldsymbol{*} \pi_{3 ;+4 / 3,-5 / 3}=\frac{1}{3}\left\{\pi_{3 ; 0,-3}+\underline{2 \pi_{3 ;+2,-1}}\right\} \\
& =\frac{1}{3} \operatorname{diag}\{, 16,13,10, \quad 7,4, \quad \underline{1},-2,-5, \quad-8,-11,-14,,\}(49 .-4) \\
& \pi_{3 ;+7 / 6,-11 / 6}=\frac{1}{3}\left\{\pi_{3 ;-1 / 2,-7 / 2}+2 \pi_{3 ;+2,-1}\right\} \\
& =\frac{1}{6} \operatorname{diag}\{, 31,25, \quad 19, \quad 13,7, \quad 1,-5,-11,-17,-23,-29,\} \quad(49 .-5) \\
& \Delta \pi_{3 ;+1,-2}=\frac{1}{3}\left\{\pi_{3 ;-1,-4}+\underline{2 \pi_{3 ;+2,-1}}\right\} \\
& =\operatorname{diag}\{, 5, \quad 4, \quad 3, \quad 2, \quad 1, \quad \underline{0}, \quad-1, \quad-2, \quad-3, \quad-4, \quad-5,,\} \quad(49 .-6) \\
& \pi_{3 ;+5 / 6,-1 / 2}=\frac{1}{3}\left\{\pi_{3 ;-3 / 2,-9 / 2}+\underline{2 \pi_{3 ;+2,-1}}\right\} \\
& =\frac{1}{6} \operatorname{diag}\{, 29, \quad 23,17, \quad 11,5, \quad-1,-7,-13,-19,-25,-31,,\}(49 .-7) \\
& \text { * } \pi_{3 ;+2 / 3,-7 / 3}=\frac{1}{3}\left\{\pi_{3 ;-2,-5}+\underline{2 \pi_{3 ;+2,-1}}\right\} \\
& =\frac{1}{3} \operatorname{diag}\{, 14, \quad 11, \quad 8, \quad 5, \quad 2, \quad \underline{-1},-4,-7,-10,-13,-16,,\} \quad(49 .-8) \\
& \diamond \pi_{3 ;+1 / 2,-5 / 2}=\frac{1}{3}\left\{\pi_{3 ;-5 / 2,-11 / 2}+2 \pi_{3 ;+2,-1}\right\} \\
& =\frac{1}{2} \operatorname{diag}\{, \quad 9, \quad 7, \quad 5, \quad 3, \quad 1, \quad \underline{-1}, \quad-3,-5, \quad-7, \quad-9,-11,,\} \quad(49 .-9) \\
& \text { * } \pi_{3 ;+1 / 3,-8 / 3}(1)=\frac{1}{3}\left\{\pi_{3 ;-3,-6}+\underline{2 \pi_{3 ;+2,-1}}\right\} \\
& \frac{1}{3} \operatorname{diag}\{, \quad 13, \quad 10, \quad 7, \quad 4, \quad 1, \quad \underline{-2}, \quad-5,-8,-11,-14,-17,,\}(49 .-10) \\
& \pi_{3 ;+1 / 6,-17 / 6}=\frac{1}{3}\left\{\pi_{3 ;-7 / 2,-13 / 2}+\underline{2 \pi_{3 ;+2,-1}}\right\} \\
& =\frac{1}{6} \operatorname{diag}\{, 25,19, \quad 13, \quad 7, \quad 1, \quad \underline{-5},-11,-17,-23,-29,-35,,\}(49 .-11) \\
& \diamond \pi_{3 ; 0,-3}=\frac{1}{3}\left\{\pi_{3 ;-4,-7}+\underline{2 \pi_{3 ;+2,-1}}\right\} \\
& =\operatorname{diag}\{, 4, \quad 3, \quad 2, \quad 1, \quad 0, \quad-1, \quad-2, \quad-3, \quad-4, \quad-5, \quad-6,,\}(49 .-12)
\end{aligned}
$$

By the way, the intervals between above two adjoining $\pi_{3}$ is $\frac{\hbar}{6}$

$$
\Delta \pi_{3 ; m / 6+2, m / 6-1}=\pi_{3 ;(m+1) / 6+2,(m+1) / 6-1}-\pi_{3 ; m / 6+2, m / 6-1}=\frac{\hbar}{6} I_{0}
$$

Reducing (30), (47), (50) to following limitation

$\operatorname{Lim}_{n \rightarrow \infty} \Delta \pi_{3 ; m / 2 n+2, m / 2 n-1}=\pi_{3 ;(m+1) / 2 n+2,(m+1) / 2 n-1}-\pi_{3 ; m / 2 n+2, m / 2 n-1}=\frac{\hbar}{2 n} I_{0}$

And $\pi_{+4,-5}^{2}(3)=\pi_{m+2, m-1}^{2}(1)=\pi_{m / 6+2, m / 6-1}^{2}(1)=1(1+1) I_{0} \hbar^{2}=2 I_{0} \hbar^{2}$

\section{Conclusions}

This paper bases on the principle of the addition of spin angular momentums in STS frame, trying to explain the Non-boson-spinization phenomenon of light photon that occurred in [1]. Particle's spin angular momentums itself, which are influencing on the light photon interference, maybe, rather than the physical quantity phase of propagating light wave causing alone, from previous experiences.

By Table A. Explanation for what happening in photonic fermionization of light photon experiment [1].

By Table B. Suggestion for dectecting the possible existence of photonic one-third-spinization phenomenon of light photon, by using three beams of light photon in interference experiment.

By (51) When the numbers of beams of light photon increase, the intervals between two adjoining $\pi_{3}$ become narrower, and the interference patterns approach to continuous spectrum. 
Table A. Interference by two beams of light photon [1] fermionization phenomenon of light photon

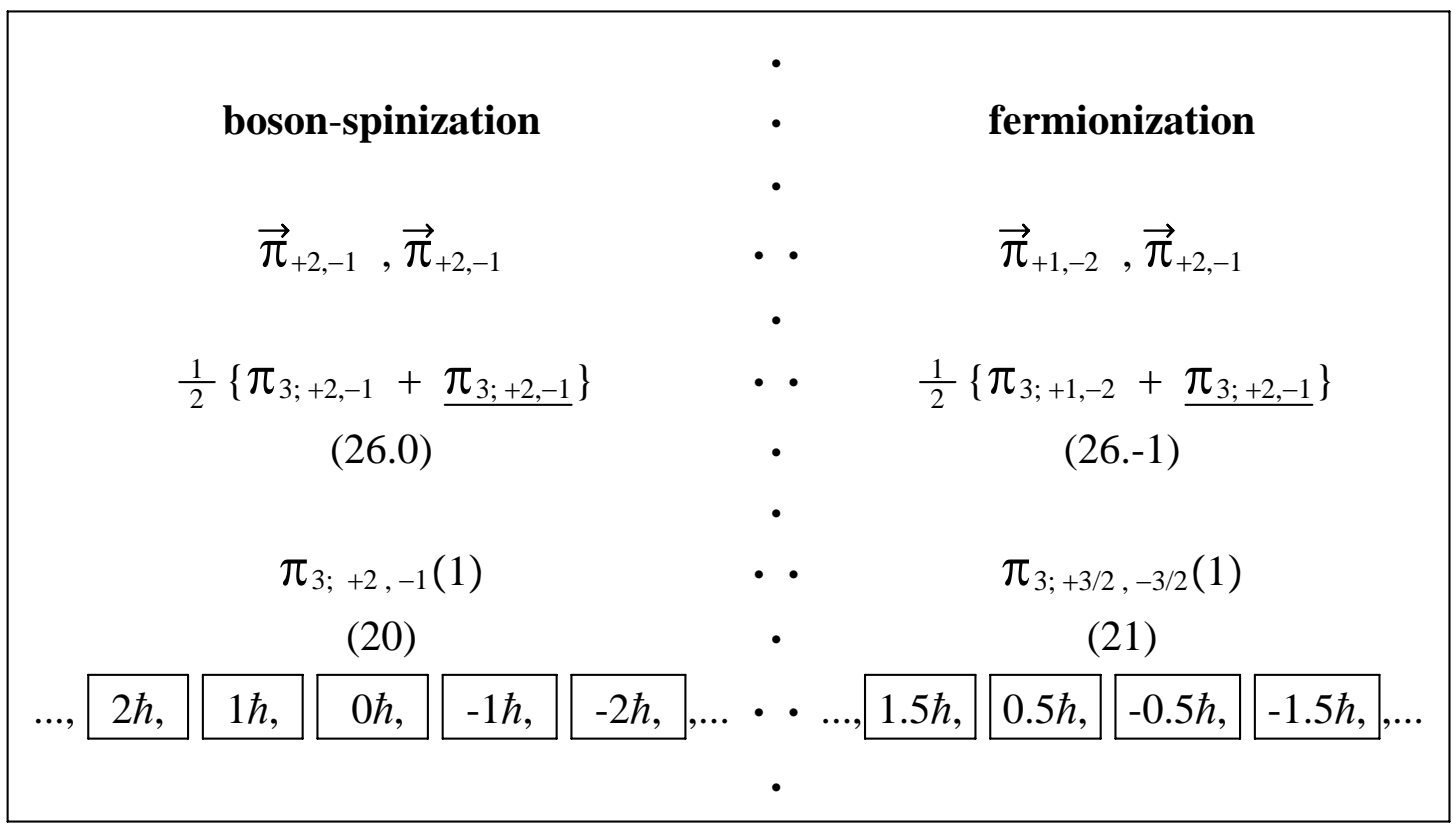

Table B. Interference by three beams of light photon one-third-spinization phenomenon of light photon

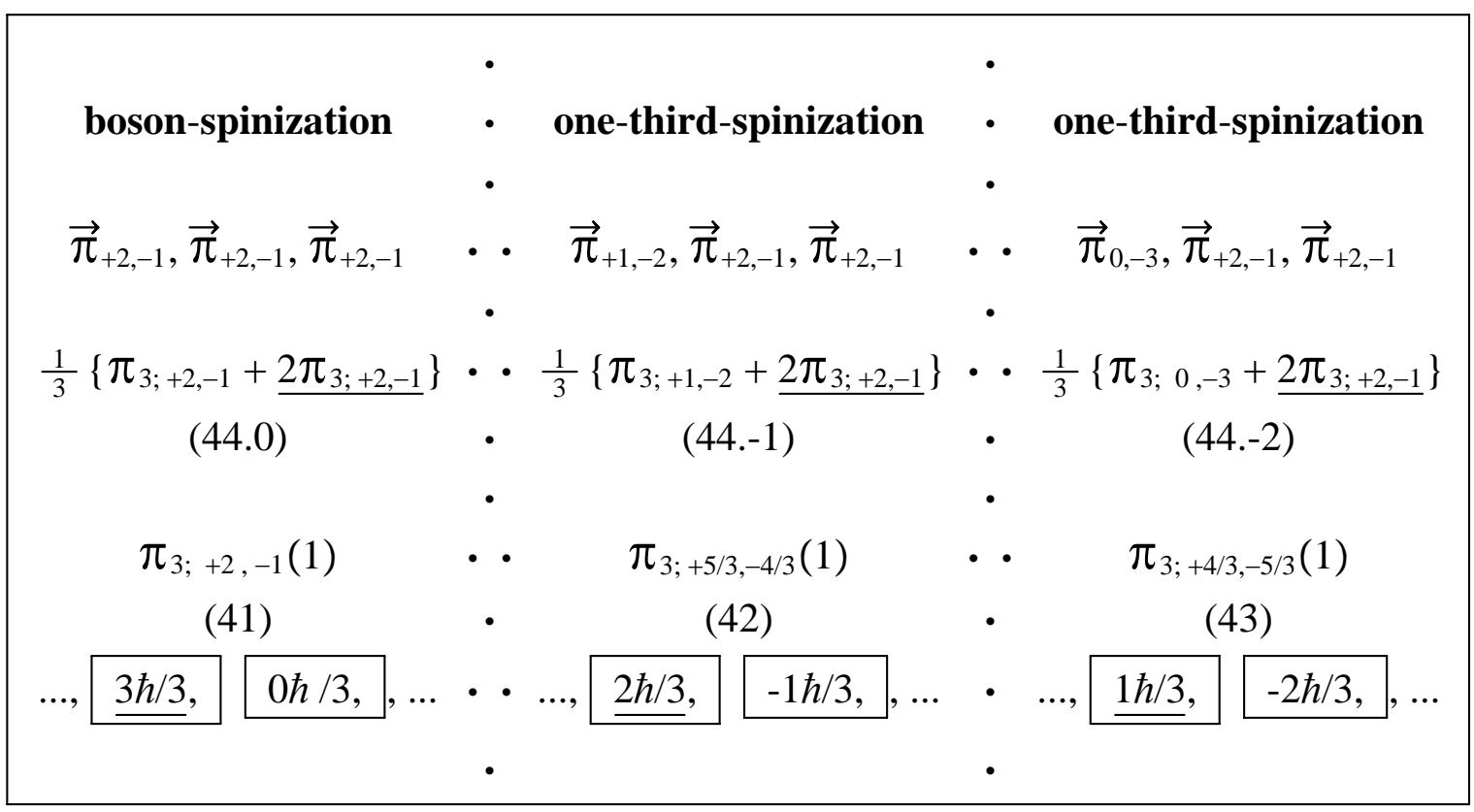




\section{References}

[1] Kyle E. Ballantine, John F. Donegan and Paul R. Eastham Science Advances 29 Apr 2016: Vol.2, No.4, e1501748 DOI: $10.1126 /$ sciadv.1501748

[2] ShaoXu Ren The Origins of Spins of Elementary Particles ISBN 9 78-988-13649-7-5 (2014);

Journal of Modern Physics, 5, 1848-1879

http:/dx.doi.org/10.4236/jmp.2014.517181;

Interaction of the Origins of Spin Angular Momentum ISBN 978-988-14902-0-9 (2016 2nd edition);

Journal of Modern Physics, 7, 737-759 http:/dx.doi.org/10.4236/jmp.2016.78070

[3] ShaoXu Ren The Third Kind of Particles ISBN 978-7-900500-91-5 (2011), ISBN 978-988-15598-9-0 (2012), ISBN 988-3-659-17892-4 (2012);

Journal of Modern Physics, 5, 800-869 http:/dx.doi.org/10.4236/jmp.2014.59090 


\section{Submit or recommend next manuscript to SCIRP and we will provide best service for you:}

Accepting pre-submission inquiries through Email, Facebook, LinkedIn, Twitter, etc.

A wide selection of journals (inclusive of 9 subjects, more than 200 journals)

Providing 24-hour high-quality service

User-friendly online submission system

Fair and swift peer-review system

Efficient typesetting and proofreading procedure

Display of the result of downloads and visits, as well as the number of cited articles

Maximum dissemination of your research work

Submit your manuscript at: http://papersubmission.scirp.org/ 